\title{
Bayesian Network Structure Learning by Recursive Autonomy Identification
}

\author{
Raanan Yehezkel and Boaz Lerner \\ Pattern Analysis and Machine Learning Lab \\ Department of Electrical \& Computer Engineering \\ Ben-Gurion University, Beer-Sheva 84105, Israel \\ \{raanany, boaz\}@ee.bgu.ac.il
}

\begin{abstract}
We propose the recursive autonomy identification (RAI) algorithm for constraint-based Bayesian network structure learning. The RAI algorithm learns the structure by sequential application of conditional independence (CI) tests, edge direction and structure decomposition into autonomous substructures. The sequence of operations is performed recursively for each autonomous sub-structure while simultaneously increasing the order of the CI test. In comparison to other constraint-based algorithms d-separating structures and then directing the resulted undirected graph, the RAI algorithm combines the two processes from the outset and along the procedure. Thereby, learning a structure using the RAI algorithm requires a smaller number of high order CI tests. This reduces the complexity and run-time as well as increases structural and prediction accuracies as demonstrated in extensive experimentation.
\end{abstract}

\section{Introduction}

A Bayesian network $(\mathrm{BN})$ is a graphical model that efficiently encodes the joint probability distribution for a set of variables [1]. Learning the model structure from data by considering all possible structures exhaustively is infeasible as the number of possible structures grows exponentially with the number of nodes [2]. Hence, structure learning requires either sub-optimal heuristic search algorithms or algorithms which are efficient under certain assumptions. In the constraint-based (CB) approach [3], [4], a structure edge is learned if meeting a constraint, called conditional independence (CI) test, derived from comparing the value of a statistical or information-theory-based test of conditional independence to a threshold. Meeting such constraints enables the formation of an undirected graph that is further directed based on causality inference rules [5]. Once the structure is learned, the model parameters are usually computed from the relative frequencies of variable states as represented in the data.

Most of the CB algorithms, such as IC [5], PC [4] and TPDA [3], construct a BN in two stages. The first stage is learning associations between variables for constructing an undirected structure. This requires an exponentially growing number of CI tests with the number of nodes. The PC and TPDA algorithms reduce the number of CI tests using some assumptions to restrict the space of possible structures. The second 
stage in most algorithms is directing edges using inferred causality performed in two steps: finding and directing $\mathrm{V}$-structures and inductively directin additional edges [5]. Edge direction is unstable, i.e., small errors in the input to the stage yield large errors in its output [4]. To eliminate the instability, the algorithms separate the two stages trying to minimize in the first stage erroneous decisions about independence caused by large condition sets that are more likely to be incorrect and also lead to poorer estimation of dependences due to the curse-of-dimensionality.

We propose the recursive autonomy identification (RAI) algorithm, which is a $\mathrm{CB}$ model that learns the structure of a BN by sequential application of CI tests, edge direction and structure decomposition into autonomous sub-structures. This sequence of operations is performed recursively for each autonomous sub-structure. In each recursive call, the order of the CI test is increased similarly to the PC algorithm [4]. By performing CI tests of low order (i.e., tests employing small conditions sets) before those of high order, the RAI algorithm performs more reliable tests that save performing less reliable tests. By considering directed rather than undirected edges, the RAI algorithm avoids unnecessary CI tests and performs tests using smaller condition sets. Smaller condition sets are represented more precisely than larger sets in the dataset, i.e., the RAI algorithm diminishes the curse-ofdimensionality while testing using smaller condition sets and thereby it improves the prediction accuracy. Repeated recursively for autonomies decomposed from the graph, both mechanisms reduce computational and time complexities, database queries and structural errors.

Section 2 provides preliminaries and Section 3 introduces the RAI algorithm. Section 4 presents experimental evaluation of the RAI algorithm regarding structural correctness, complexity and prediction accuracy. Section 5 summarizes the study.

\section{Preliminaries}

A BN $B(G, \Theta)$ is a model for representing the joint probability distribution for a set of variables $\boldsymbol{X}=\left\{X_{1}, \ldots, X_{n}\right\}$. The structure $\mathrm{G}(\mathbf{V}, \mathbf{E})$ is a directed acyclic graph (DAG) composed of $\mathbf{V}$, a set of nodes representing the variables $\boldsymbol{X}$, and $\mathbf{E}$, a set of directed edges connecting the nodes. An edge manifests dependence between the nodes connected by the edge while the absence of an edge demonstrates independence between the nodes. A directed edge $X_{i} \rightarrow X_{j}$ connects a child node $X_{j}$ to its parent $X_{i} \cdot \boldsymbol{P a}(X, \mathrm{G})$ is the set of $X$ 's parents in $\mathrm{G}$. The set of parameters $\Theta$ holds local conditional probabilities over $\boldsymbol{X}, P\left(X_{i} \mid \boldsymbol{P a}\left(X_{i}, \mathrm{G}\right)\right) \forall i$ that quantify the edges. The joint probability distribution for $\boldsymbol{X}$ represented by a $\mathrm{BN}$ is $P\left(X_{1}, \ldots X_{n}\right)=\prod_{i=1}^{n} P\left(X_{i} \mid \boldsymbol{P a}\left(X_{i}, G\right)\right)[1]$. We also use the term partially directed graph (PDG), i.e., a graph which may have both directed and undirected edges and has at most one edge between any pair of nodes. We use this term in learning a graph starting from a complete undirected graph and eliminating and directing edges until uncovering a graph representing a family of Markov equivalent structures (pattern) of the true BN (i.e., the graphs have the same sets of adjacencies and V-structures) [4], [5]. $\boldsymbol{P a}(X, \mathrm{G}), \boldsymbol{A d j}(X, \mathrm{G})$ and $\boldsymbol{C h}(X, \mathrm{G})$ are respectively the sets of potential parents, adjacent nodes (two nodes connected by an edge) and children of $X$ in a 
PDG, $\boldsymbol{P a} \boldsymbol{a}_{p}(X, \mathrm{G})=\boldsymbol{A d j}(X, \mathrm{G}) \backslash \boldsymbol{C h}(X, \mathrm{G})$. We use $X \| Y \mid \boldsymbol{S}$ to indicate that $X$ and $Y$ are independent given a set of nodes $S$ and employ also the notion of d-separation [5]. Next, we define $\mathrm{d}$-separation resolution evaluating $\mathrm{d}$-separation for different sizes of condition sets, an exogenous cause to a graph and an autonomous sub-structure.

Definition 1 - $d$-separation resolution: The resolution of a d-separation relation between a pair of non-adjacent nodes in a graph is the size of the smallest condition set that d-separates the two nodes (see Figure 1 for an example).

Definition 2 - $d$-separation resolution of a graph: The d-separation resolution of a graph is the highest d-separation resolution in the graph (see Figure 2).

(a)

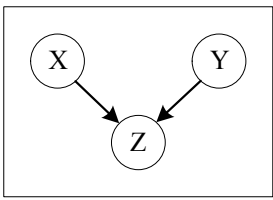

(b)

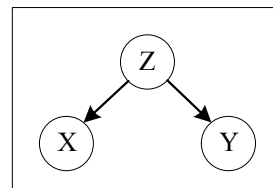

(c)

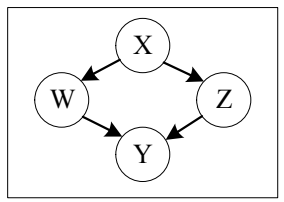

Fig. 1. Examples of d-separation resolutions of (a) 0, (b) 1 and (c) 2 between nodes $X$ and $Y$

(a)

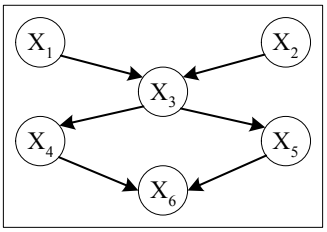

(b)

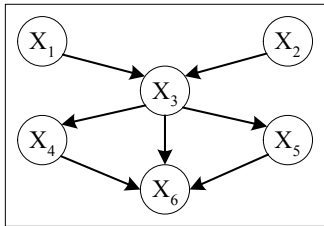

(c)

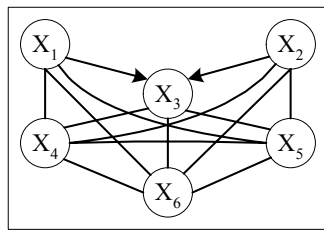

Fig. 2. Examples of graph d-separation resolutions of (a) 2, (b) 1 and (c) 0

Definition 3 - exogenous cause: $Y$ is an exogenous cause to $G(\mathbf{V}, \mathbf{E})$ if $Y \notin \mathbf{V}$ and $\forall X \in \mathbf{V}, Y \in \boldsymbol{P a}(X)$ or $Y \notin \operatorname{Adj}(X)[5]$.

Definition 4 - autonomous sub-structure: A sub-structure $\mathrm{G}^{\mathrm{A}}\left(\mathbf{V}^{\mathbf{A}}, \mathbf{E}^{\mathbf{A}}\right)$ in $\mathrm{G}(\mathbf{V}, \mathbf{E})$ s.t $\mathbf{V}^{\mathbf{A}} \subset \mathbf{V}, \mathbf{E}^{\mathbf{A}} \subset \mathbf{E}$ is autonomous given a set $\mathbf{V}_{\text {ex }}$ of exogenous nodes to $\mathrm{G}^{\mathrm{A}}$ if $\forall X \in \mathbf{V}^{\mathbf{A}}$, $\boldsymbol{P a}(X, \mathrm{G}) \subset\left\{\mathbf{V}^{\mathbf{A}} \cup \mathbf{V}_{\mathrm{ex}}\right\}$. If $\mathbf{V}_{\mathrm{ex}}$ is empty, we say that the sub-structure is autonomous.

An autonomous sub-structure $\mathrm{G}^{\mathrm{A}}$ holds the Markov property, i.e., two non-adjacent nodes in $\mathrm{G}^{\mathrm{A}}$ are d-separated given nodes in $\mathrm{G}^{\mathrm{A}}$ or exogenous causes to $\mathrm{G}^{\mathrm{A}}$ (Figure 3 ).

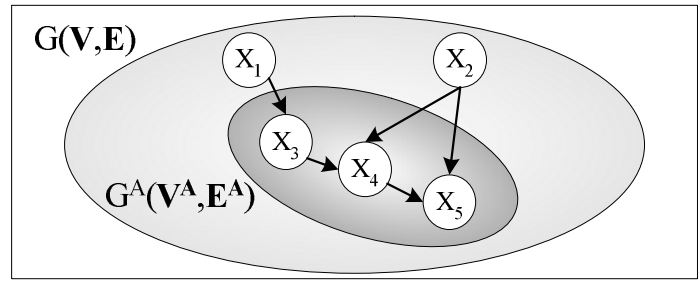

Fig. 3. An autonomous sub-structure $\mathrm{G}^{\mathrm{A}}$ in $\mathrm{G}$ having exogenous nodes $X_{1}$ and $X_{2}$ 


\section{Recursive Autonomy Identification}

Starting from a complete undirected graph and proceeding from low to high graph dseparation resolution, the RAI algorithm uncovers the correct pattern of a structure by recursively performing the sequence: (1) test of CI between nodes followed by the removal of edges related to independences, (2) edge direction according to inferred causality rules, and (3) graph decomposition into autonomous sub-structures.

CI testing of order $n$ between nodes $X$ and $Y$ is performed by thresholding the value of a criterion measuring the dependence between the nodes conditioned on a set of $n$ nodes (the condition set) from the parents of $X$ or $Y$. The set is determined by the Markov property [5], i.e., if $X$ is directed into $Y$ then only $Y^{\prime}$ 's parents are included in the set. Commonly, this criterion is the $\chi^{2}$ goodness of fit test [4] or conditional mutual information [3]. Directing edges is conducted according to causality rules [5]. Given an undirected graph and a set of independences, the following two steps are performed consecutively. First, V-structures are identified and the corresponding edges are directed. A V-structure $X \rightarrow Z \leftarrow Y$ is defined if 1) $X$ and $Y$ are unconnected neighbors of $Z, 2$ ) $X$ and $Y$ are marginally independent and 3) $X$ and $Y$ are dependent conditioned on $Z$. In the second step, also called the inductive stage, an edge $Y-Z$ is directed as $Y \rightarrow Z$ if: 1) there exists an edge $X \rightarrow Y$ where $X$ and $Z$ are not adjacent and there is no arrowhead at $Y, 2$ ) there is a chain $Y \rightarrow X \rightarrow Z$, or 3) two chains $Y-X \rightarrow Z$ and $Y-W \rightarrow Z$ exist. This step is continued until no more edges can be directed complying with two restrictions: a) no $\mathrm{V}$-structures in addition to those of the first step and b) no directed cycles are created. Finally, decomposition into autonomous sub-structures reveals the structure hierarchy. It also allows the performance of fewer CI tests that are conditioned on a large number of potential parents, and hence reduces complexity. The RAI algorithm identifies ancestor and descendant sub-structures; the formers are autonomous and the latters are autonomous given nodes of the formers.

An iteration of the RAI algorithm starts with knowledge produced in the previous iteration and the current d-separation resolution, $n$. Previous knowledge includes $G_{\text {start }}$, a structure having d-separation resolution of $n-1$, and $\mathbf{G}_{\mathrm{ex}}$, a set of structures having each possible exogenous causes to $\mathrm{G}_{\text {start }}$. In the first iteration, $n=0, \mathrm{G}_{\text {start }}(\mathbf{V}, \mathbf{E})$ is the complete undirected graph and $\mathbf{G}_{\mathrm{ex}}=\varnothing$. Given a structure $\mathrm{G}_{\text {start }}$ having d-separation resolution $n$ - 1 , the RAI algorithm seeks independences between adjacent nodes conditioned on sets of size $n$, resulting in a structure having d-separation resolution of $n$. After applying causality rules in order to direct the edges, a partial topological ordering is obtained in which parent nodes precede their descendants. This ordering is partial as not all the edges can be directed, so nodes connected by undirected edges have equal topological order. Using this partial topological ordering, the algorithm decomposes the structure into ancestor and descendent autonomous sub-structures in order to reduce the complexity of the successive stages. A descendant sub-structure is established by identifying the lowest topological order nodes (either a single node or a several nodes having the same lowest order). We will refer to a single descendent sub-structure although it may consist of a several non-connected sub-structures. This structure is autonomous given nodes of higher topological order composing ancestor sub-structures. The algorithm first learns ancestor sub-structures and only then the descendant sub-structure in order to consider for each pair of nodes of the descendent sub-structure condition sets that (possibly) have 
smaller numbers of parents. Each ancestor or descendent sub-structure is further learned by recursive calls to the algorithm. Figures 4 and 5 show respectively the RAI algorithm and a manifesting example.

The RAI algorithm is composed of four stages (A to D in Figure 4) and an exit condition checked before the execution of each stage. The purpose of Stage $\mathrm{A}$ is to shrink the link between $\mathbf{G}_{\mathrm{ex}}$ and $\mathrm{G}_{\text {start }}$, the latter is having d-separation resolution of $n-1$, and direct the edges of $\mathrm{G}_{\text {start }}$. This is achieved by CI testing using condition sets of size $n$ between nodes in $\mathbf{G}_{\text {ex }}$ and nodes in $\mathrm{G}_{\text {start }}$, removing edges corresponding to independences and directing those remaining edges that can be directed. Stage B performs the same for edges in $G_{\text {start }}$. In both stages, the condition set includes nodes of $\mathbf{G}_{\mathrm{ex}}$ and $\mathrm{G}_{\text {start }}$. Stage B also identifies the partial topological ordering of nodes and decomposes the current graph into ancestor and descendant substructures. Stage C is a recursive call to the RAI algorithm for learning each ancestor sub-structure with order $n+1$. Similarly, Stage D of the algorithm is a recursive call to the RAI for learning the descendant sub-structure with order $n+1$ while assuming that the ancestor sub-structures have been fully learned (having the maximal d-separation resolution).

Figure 5 sketches stages in learning an example graph. Figure 5a shows the true structure we wish to uncover. Initially, $\mathrm{G}_{\text {start }}$ is the complete undirected graph, $n=0$ and $\mathbf{G}_{\mathrm{ex}}$ is empty so Stage A is skipped. In Stage B1, pairs of nodes are CI tested given empty condition sets i.e., marginal independence, which yields the removal of the edges between node $X_{1}$ and nodes $X_{3}, X_{4}$ and $X_{5}$ (Figure 5b). The causal relations inferred in Stage B2 are shown in Figure 5c. The nodes having the lowest topological order $\left(X_{2}, X_{6}, X_{7}\right)$ are grouped into a descendant sub-structure $\mathrm{G}_{\mathrm{D}}$ (Stage B3) while the remaining nodes form two unconnected ancestor sub- structures, $\mathrm{G}_{\mathrm{A}_{1}}$ and $\mathrm{G}_{\mathrm{A}_{2}}$ (Stage B4) (Figure 5d). In Stage C, the algorithm is called recursively for each of the ancestor sub-structures with $n=1, \mathrm{G}_{\text {start }}=\mathrm{G}_{\mathrm{A}_{\mathrm{i}}}$ and $\mathbf{G}_{\mathrm{ex}}=\varnothing$. Since sub-structure $\mathrm{G}_{\mathrm{A}_{1}}$ contains a single node, the exit condition for the structure is satisfied. While calling $\mathrm{G}_{\text {start }}=\mathrm{G}_{\mathrm{A}_{2}}$, Stage A is skipped and Stage B1 identifies that $X_{4} \| X_{5} \mid X_{3}$ thus removes $X_{4}-X_{5}$. No causal relations are identified so the nodes have equal topological order and they are grouped to from a descendant sub-structure. The recursive call for this sub-structure with $n=2$ is returned immediately since the exit condition is satisfied (Figure 5e). In Stage D, the RAI is called with $n=1, \mathrm{G}_{\mathrm{start}}=\mathrm{G}_{\mathrm{D}}$ and $\mathbf{G}_{\mathrm{ex}}=\left\{\mathrm{G}_{\mathrm{A}_{1}}, \mathrm{G}_{\mathrm{A}_{2}}\right\}$. In Stage A1 relations $X_{1} \Perp \underline{\|}\left\{X_{6}, X_{7}\right\}\left|X_{2}, X_{4} \|\left\{X_{6}, X_{7}\right\}\right| X_{2}$ and $\left\{X_{3}, X_{5}\right\} \|\left\{X_{2}, X_{6}, X_{7}\right\} \mid X_{4}$ are identified and the corresponding edges are removed (Figure 5f). In Stage A2, $X_{2}$ is identified as a parent of $X_{6}$ and $X_{7}$ (Figure $5 \mathrm{~g}$ ). Stage B1 identifies that $X_{2} \| X_{7} \mid X_{6}$ and Stage B2 identifies $X_{6}$ as a parent of $X_{7}$ leading, respectively, to the removal of $X_{2} \rightarrow X_{7}$ and direction of $X_{6} \rightarrow X_{7}$ (Figure 5h). Then, in Stages B3 and B4, $X_{7}$ and $\left\{X_{2}, X_{6}\right\}$ are identified as a descendant and an ancestor sub-structures, respectively. Further recursive calls are returned immediately and the resulting PDG (Figure 5h) represents a family of Markov equivalent structures of the true structure (Figure 5a). 
Main function $\mathrm{G}_{\text {out }}=\operatorname{RAI}\left(n, \mathrm{G}_{\text {start }}\left(\mathbf{V}_{\text {start }}, \mathbf{E}_{\text {start }}\right), \mathbf{G}_{\text {ex }}\left(\mathbf{V}_{\text {ex }}, \mathbf{E}_{\text {ex }}\right)\right)$

\section{Exit condition}

If all nodes in $\mathrm{G}_{\text {start }}$ have less than $n+1$ potential parents exit.

A. Thinning the link between $\mathbf{G}_{\mathrm{ex}}$ and $\mathbf{G}_{\text {start }}$ and directing $\mathbf{G}_{\text {start }}$

1. $\forall Y$ in $\mathbf{G}_{\text {start }}$ and its parent $X$ in $\mathbf{G}_{\mathbf{e x}}$, if $\exists \boldsymbol{S} \subset\left\{\boldsymbol{P a}\left(Y, \mathbf{G}_{\mathbf{e x}}\right) \backslash X \cup \boldsymbol{P} \boldsymbol{a}_{p}\left(Y, \mathrm{G}_{\text {start }}\right)\right\}$ and $|\boldsymbol{S}|=n$ s.t $X \Perp Y \mid \boldsymbol{S}$, then remove the edge between $X$ and $Y$.

2. Direct the edges using causality inference rules.

B. Thinning, directing and decomposing $\mathbf{G}_{\text {start }}$

1. $\forall Y$ and its potential parent $X$ both in $\mathrm{G}_{\text {start }}$, if $\exists \boldsymbol{S} \subset\left\{\boldsymbol{P a}\left(Y, \mathbf{G}_{\text {ex }}\right) \cup \boldsymbol{P a}\left(Y, \mathrm{G}_{\text {start }}\right) \backslash X\right\}$ and $|\boldsymbol{S}|=n$ s.t $X \Perp Y \mid \boldsymbol{S}$, then remove the edge between $X$ and $Y$.

2. Direct the edges using causality inference rules.

3. Group nodes having the lowest topological order into a descendant sub-structure $G_{D}$.

4. Remove $G_{D}$ from $G_{\text {start }}$ temporarily and define the resulting unconnected structures as ancestor sub-structures $G_{A_{1}}, \ldots, G_{A_{k}}$.

\section{Ancestor sub-structure decomposition} for $i=1$ to $k$, call $\mathrm{RAI}\left(n+1, \mathrm{G}_{\mathrm{A}_{i}}, \mathbf{G}_{\mathrm{ex}}\right)$

\section{Descendant sub-structure decomposition}

1. Define $\mathbf{G}_{\mathrm{D}_{-} \mathrm{ex}}=\left\{\mathrm{G}_{\mathrm{A}_{1}}, \ldots, \mathrm{G}_{\mathrm{A}_{\mathrm{k}}}, \mathbf{G}_{\mathrm{ex}}\right\}$ as the exogenous structure to $\mathrm{G}_{\mathrm{D}}$.

2. Call $\operatorname{RAI}\left(n+1, \mathrm{G}_{\mathrm{D}}, \mathbf{G}_{\mathrm{D}_{-} \mathrm{ex}}\right)$

Fig. 4. The RAI algorithm

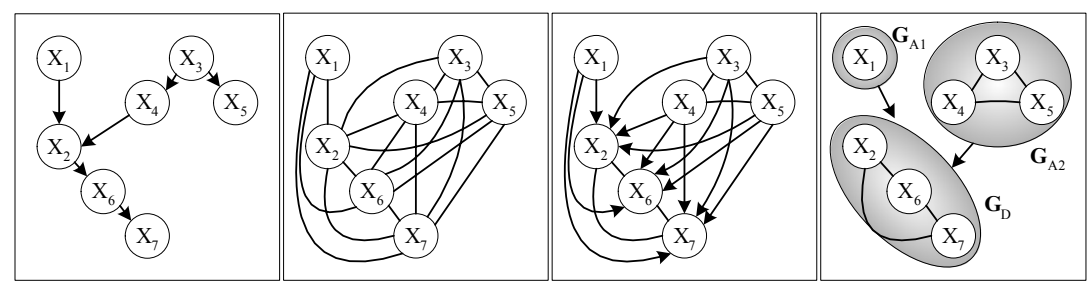

(a)

(b)

(c)

(d)

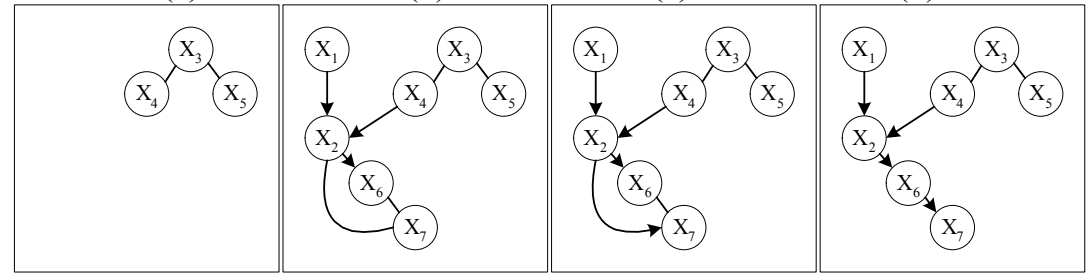

(e)

(f)

(g)

(h)

Fig. 5. Learning an example structure. a) The true structure and structures learned in Stages (see Figure 4) b) B1, c) B2, d) B3 and B4, e) C, f) D and A1, g) D and A2 and h) D, B1 and B2.

\section{Experiments and Results}

Synthetic data. The complexity of the RAI algorithm was compared to that of the PC algorithm by the number of CI tests required to learn synthetically generated 
structures. We learned graphs of sizes (numbers of nodes) between 6 and 15 . We used 3,000 randomly generated graphs restricted by a maximal fan-in value of 3 , i.e., every node has at most 3 parents and at least one node has 3 parents. The implementation was aided by the Bayes net toolbox (BNT) [6] and BNT structure learning package [7]. Figure 6a shows the percentage of CI tests saved using the RAI algorithm compared to the PC algorithm as a function of the condition set size for different graph sizes. The figure shows that the percentage of CI tests saved using the RAI algorithm increases with both graph and condition set sizes. For example, the save in CI tests for a graph of size 15 and condition sets of size 4 is more than $70 \%$.

ALARM network. Correctness of the learned structure was evaluated using the ALARM network [8], which is a widely accepted structure learning benchmark since the true graph is known. The RAI algorithm was compared to the PC, TPDA and K2 [2] algorithms using 10 databases of 10,000 randomly generated cases each. The node ordering required for the $\mathrm{K} 2$ algorithm was determined by learning a maximum weighted spanning tree (MWST) and selecting a root node [7]. We also evaluated the $\mathrm{K} 2$ algorithm with the true ordering, which is inferred from the known network. We identify these two versions as K2 (MWST) and K2 (true), respectively. Since the TPDA algorithm uses for CI testing the conditional mutual information (CMI) [3], we employ this test also for the RAI and PC algorithms and selected thresholds of $3 \cdot 10^{-3}$, $3 \cdot 10^{-3}$ and $2 \cdot 10^{-3}$ for the RAI, TPDA and PC algorithms, respectively. Structural correctness was evaluated by measuring the root mean square of extra and missing edges errors in the learned structure compared with the true structure (i.e., the total error). The smallest total error of $1.3 \%$ was achieved by the RAI algorithm compared to errors of $6.32 \%, 2.94 \%, 6.76 \%$ and $4.68 \%$ of the TPDA, PC, K2 (MWST) and K2 (true) algorithms, respectively. This superiority of the RAI algorithm was validated using a $t$-test with $1 \%$ significance level. Complexity was measured by the total number of log operations (logarithms, multiplications and divisions) required for calculating CMI in CI testing. As Figure 6b shows, the PC and TPDA algorithms require respectively, 521\% and 394\% more log operations than the RAI algorithm.

Real-world data. The RAI prediction accuracy was evaluated using databases of the UCI Repository [9]. Continuous variables were discretized and instances with missing values were removed. All databases were analyzed using a CV5 experiment except the large "chess", "mofn 3-7-10", "nursery" and "shuttle" databases which were analyzed using the holdout method. CI tests were carried out using the $\chi^{2}$ test, which is recommended for the PC algorithm [4], with thresholds chosen for each algorithm and database in order to maximize the prediction accuracy on a validation set. Parameter learning was performed assuming Dirichlet prior distribution having zero hyperparameters leading to the maximum likelihood solution [1]. Prediction accuracies of the RAI and PC algorithms as well as the run-time saved by using the RAI are summarized in Table 1. The accuracy is also compared to those of the TPDA algorithm [10] and three classifiers reported in [11], namely, the naïve Bayesian classifier (NBC), tree augmented naïve (TAN) Bayes and a BN learned using the minimum description length (MDL) score. Databases in Table 1 for which accuracies are not reported in [10] or [11] are represented by empty entries. On thirteen of the nineteen databases, the RAI algorithm improves accuracy on the PC algorithm, on five it keeps accuracy intact and on the remaining "iris" database it deteriorates accuracy. Since 


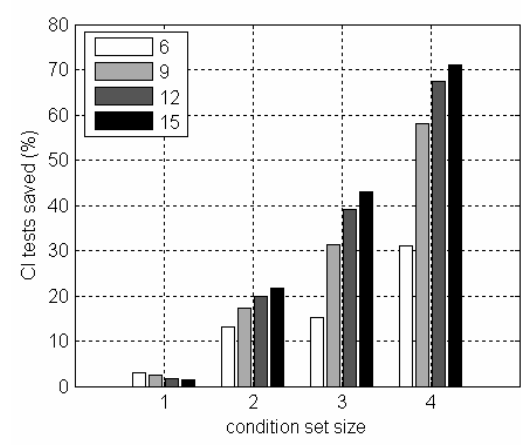

(a)

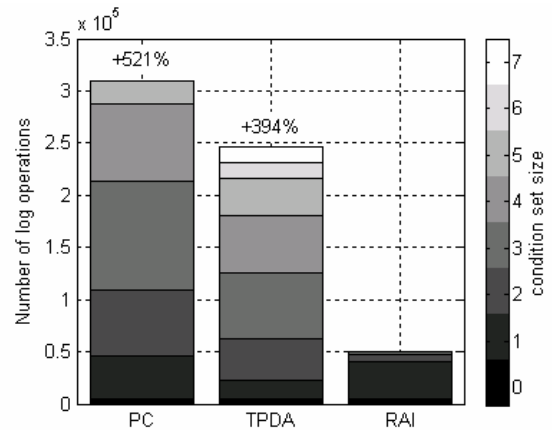

(b)

Fig. 6. (a) Percentage of CI tests saved by using the RAI algorithm compared to the PC algorithm as a function of the condition set size and number of nodes in the graph $-6,9,12$ or 15 (gray shades). (b) The numbers of log operations required by the PC, TPDA, and RAI algorithms for learning the ALARM network. Gray shades represent different condition set sizes for the CI tests. Percentages on the tops of the bars are with reference to the RAI algorithm.

Table 1. Mean prediction accuracy of classifiers based on the RAI, PC, TPDA, NBC, MDL and TAN. Bold font emphasizes the highest accuracy for a database. Also shown is the run-time cut due to the RAI compared to the PC algorithm. Standard deviationa are ommited.

\begin{tabular}{|c|c|c|c|c|c|c|c|}
\hline Database & $\begin{array}{c}\text { RAI } \\
(\%)\end{array}$ & $\begin{array}{c}\text { PC } \\
(\%)\end{array}$ & $\begin{array}{c}\text { TPDA } \\
(\%)\end{array}$ & $\begin{array}{c}\text { NBC } \\
(\%)\end{array}$ & $\begin{array}{c}\text { MDL } \\
(\%)\end{array}$ & $\begin{array}{c}\text { TAN } \\
(\%)\end{array}$ & $\begin{array}{c}\text { Run-time } \\
\text { cut } \\
(\%)\end{array}$ \\
\hline australian & 85.51 & 85.51 & & $\mathbf{8 6 . 2 3}$ & $\mathbf{8 6 . 2 3}$ & 81.3 & $\mathbf{6 . 0 5}$ \\
\hline breast & 96.49 & 95.46 & & $\mathbf{9 7 . 3 6}$ & 96.92 & 95.75 & $\mathbf{7 1 . 8 7}$ \\
\hline car & $\mathbf{9 2 . 9 4}$ & 85.07 & 86.11 & & & & $\mathbf{9 1 . 1 0}$ \\
\hline chess & 93.53 & 93.15 & 94.65 & 87.15 & $\mathbf{9 5 . 5 9}$ & 92.40 & $\mathbf{8 0 . 6 5}$ \\
\hline cleve & 81.41 & 76.67 & & $\mathbf{8 2 . 7 6}$ & 81.39 & 79.06 & $\mathbf{3 9 . 6 0}$ \\
\hline cmc & $\mathbf{5 1 . 1 2}$ & 50.92 & & & & & $\mathbf{1 4 . 2 2}$ \\
\hline corral & $\mathbf{9 8 . 5 2}$ & 84.53 & & 85.88 & 97.60 & 95.32 & $\mathbf{8 7 . 9 4}$ \\
\hline crx & $\mathbf{8 6 . 3 8}$ & $\mathbf{8 6 . 3 8}$ & & 86.22 & 85.60 & 83.77 & $\mathbf{2 5 . 2 5}$ \\
\hline flare C & $\mathbf{8 4 . 3 0}$ & $\mathbf{8 4 . 3 0}$ & 82.27 & 79.46 & 82.74 & 82.74 & $\mathbf{2 0 . 3 8}$ \\
\hline iris & 93.33 & $\mathbf{9 6 . 0 0}$ & & 93.33 & 94.00 & 93.33 & $\mathbf{1 9 . 1 0}$ \\
\hline led7 & $\mathbf{7 3 . 5 9}$ & 73.31 & & & & & $\mathbf{9 1 . 7 4}$ \\
\hline mofn 3-7-10 & $\mathbf{9 3 . 1 6}$ & 81.45 & & 86.43 & 85.94 & 91.70 & $\mathbf{6 7 . 7 0}$ \\
\hline nursery & $\mathbf{9 3 . 0 6}$ & $\mathbf{9 3 . 0 6}$ & 89.72 & & & & $\mathbf{8 9 . 7 0}$ \\
\hline shuttle (s) & $\mathbf{9 9 . 2 2}$ & 98.40 & & 98.34 & 99.17 & 98.86 & $\mathbf{3 8 . 9 4}$ \\
\hline tic-tac-toe & $\mathbf{7 5 . 5 7}$ & 74.74 & & & & & $\mathbf{3 6 . 5 2}$ \\
\hline vehicle & $\mathbf{7 0 . 2 2}$ & 63.93 & & 58.28 & 61.00 & 67.86 & $\mathbf{1 3 . 1 5}$ \\
\hline vote & $\mathbf{9 5 . 8 7}$ & 95.64 & 95.17 & 90.34 & 94.94 & 89.20 & $\mathbf{4 6 . 0 6}$ \\
\hline wine & $\mathbf{8 7 . 0 7}$ & 85.44 & & & & & $\mathbf{2 9 . 1 1}$ \\
\hline zoo & 88.95 & 88.95 & & & & & $\mathbf{1 3 . 6 3}$ \\
\hline
\end{tabular}


the accuracies for the TPDA, NBC, MDL-based and TAN classifiers are borrowed from the original papers, no examination of statistical significance of the results could have been performed. Therefore, any advantage of a classifier over another classifier for a specific database is not necessarily statistically significant. However, averaging the prediction accuracy over the twelve databases for which we have results for all but the TPDA algorithm shows that the RAI, PC, NBC, MDL-based and TAN classifiers achieve average accuracies of $89.8,86.8,86.0,88.4$ and $87.6 \%$, respectively.

\section{Summary}

We demonstrate that the RAI algorithm requires less CI tests of high order than the PC algorithm, and the percentage of tests saved by the RAI algorithm increases with the sizes of the network and condition set for the CI test. The RAI algorithm reconstructs the ALARM network with significantly less errors than the PC, TPDA and K2 algorithms and has a considerably smaller computational complexity. In addition, the structure learned by the RAI algorithm yields a classifier that is mostly more accurate than those learned using the PC, TPDA, NBC, TAN and MDL-based algorithms.

Acknowledgment. This works was supported in part by the Paul Ivanier Center for Robotics and Production Management, Ben-Gurion University, Beer-Sheva, Israel.

\section{References}

1. Heckerman, D. A tutorial on learning with Bayesian networks. MS TR-95-06, March 1995.

2. Cooper, G. F., and Herskovits, E. A Bayesian method for the induction of probabilistic networks from data. Machine Learning, Vol. 9, 309-347, 1992.

3. Cheng, J., Bell, D. and Liu, W. Learning Bayesian networks from data: an efficient approach based on information theory. Sixth ACM Int. Conf. on Information and Knowledge Management, pages 325-331, 1997.

4. Spirtes, P., Glymour, C., and Scheines, R. Causation, Prediction and Search, 2nd edition, MIT Press, 2000.

5. Pearl, J. Causality: Models, Reasoning, and Inference. Cambridge. 2000.

6. Murphy, K. Bayes net toolbox for Matlab. Computing Science and Statistics, Vol. 33, 2001.

7. Leray, P., and Francois, O. BNT structure learning package: documentation and experiments. PSI TR, 2004.

8. Beinlich, I. A., Suermondt, H. J., Chavez, R. M., and Cooper, G. F. The ALARM monitoring system: A case study with two probabilistic inference techniques for belief networks. Second European Conf. on Artificial Intelligence in Medicine, pages 246-256, 1989.

9. Newman, D. J., Hettich, S., Blake, C. L., and Merz, C. J. UCI Repository of machine learning databases. U. of California, Irvine, Dept. of Information and Computer Science 1998.

10. Cheng, J., and Greiner, R. Comparing Bayesian network classifiers. Fifteenth Conf. on Uncertainty in Artificial Intelligence, pages 101-107, 1999.

11. Friedman, N., Geiger, D. and Goldszmidt, M. Bayesian network classifiers. Machine Learning, Vol. 29, 131-161, 1997. 